\title{
Medikamente und ihre Entwicklung für Kinder
}

\section{Klaus Rose}

Dr. med., klausrose Consulting, Pediatric Drug Development \& More, Riehen

\begin{abstract}
Wachsende Verfügbarkeit wirksamer Arzneimittel und Fortschritte der pädiatrischen klinischen Pharmakologie haben zu der Forderung geführt, Kinder verstärkt am pharmazeutischen therapeutischen Fortschritt teilhaben zu lassen. Seit 1997 gibt es eine US-pädiatrische Gesetzgebung, die der EU ist seit 2007 in Kraft. Erfüllt die EU ihre Versprechung aus 2002, «Better Medicines for Children»?
\end{abstract}

\section{Zusammenfassung}

Moderne Medikamente können Leben retten, aber auch gefährlich sein. Es bedarf einer sorgfältigen Abwägung des Benefit-Risk-Verhältnisses. Mit der Einführung moderner Arzneimittellabels begann der Hinweis, dass das jeweilige Medikament nicht für Kinder erprobt und zugelassen war. Die Kinderheilkunde ist damit lange gut zurechtgekommen, aber bereits 1968 wurde kritisch formuliert, Kinder seien «therapeutische Waisenkinder». Die Diskussion hierüber kulminierte 1997 in einer US-Gesetzgebung, die EU folgte zehn Jahre später. Seit 2007 registriert die EU neue Medikamente nur mit einem Kinderentwicklungsplan (pediatric investigation plan, PIP). EU-Versprechen wie "Better Medicines for Children» und die Forderung nach Evaluierung von Medikamenten bei Kindern erscheinen auf den ersten Blick gut. Eine kritische Analyse der PIP-Entscheidungen zeigt aber einen dogmatischen Behördenaktivismus, der systematisch sinnlose und schädliche Studien an Kindern und Jugendlichen mit seltenen und häufigen Krankheiten erzwingt. Die Beweggründe für diesen Aktivismus sind komplex, aber Parallelen zu fragwürdigen Studien der jüngeren Geschichte sind unübersehbar. Pharmazeutische Firmen müssen sich hierzu verpflichten, sonst lässt die EMA ihre neuen Medikamente nicht zu. Die Rekrutierung für diese Studien ist weltweit und macht auch vor der Schweizer Grenze nicht halt. Schweizer Ärzte, Ethikkommissionen und Eltern müssen auf der Hut sein.

1 Die Literatur zu diesem Artikel findet sich online unter www.saez.ch $\rightarrow$ Aktuelle Ausgabe oder $\rightarrow$ Archiv $\rightarrow 46$.

\section{Arzneimittel, Labels \& Kinder}

Die Thalidomid-Katastrophe mit vielen tausend Missbildungen bei Kindern zeigte erstmals weltweit, wie gefährlich Medikamente sein können. Seit 1962 verlangen die USA den Nachweis von Wirksamkeit und Sicherheit durch klinische Studien. Dies war der Beginn moderner labels (Englisch für «Etikett»). Ab jetzt wurde oft betont, dass das Medikament nicht an Kindern erprobt war. Behandlung von Kindern war ab jetzt oft off-label. 1968 prägte Shirkey den Begriff von Kindern als "therapeutischen Waisen»: nicht im Hauptfokus der Medikamentenentwicklung [1]. Ganz recht hatte er nicht. Wo ein Markt war, wurde auch für Kinder entwickelt, z.B. Impfstoffe oder Wachstumshormone. Aber oft waren Medikamente wenig für Kinder geeignet - sie können bis zum 7. Lebensjahr keine Tabletten schlucken.

\section{Pädiatrische klinische Pharmakologie und erste pädiatrische Gesetzgebung}

Bei kleinen Kindern sind Absorption, Distribution, Metabolisierung und Exkretion (ADME) oft sehr verschieden von Erwachsenen, mit der Gefahr von Unteroder Überdosierung [2]. Eine erste US-pädiatrische Gesetzgebung bot 1997 Firmen Patentverlängerung für mit der amerikanischen Zulassungsbehörde FDA abgesprochene Kinderstudien. Durch ein späteres Gesetz konnte die FDA Firmen auch zu Kinderstudien verpflichten.

\section{EU-pädiatrische Gesetzgebung}

Seit 2007 benötigt jedes neue Medikament einen von der European Medicines Agency (EMA) und seinem Pediatric Committee (PDCO) genehmigten pädiatrischen Entwicklungsplan (pediatric investigation plan, PIP). Ohne PIP wird der Zulassungsantrag für Erwachsene nicht bearbeitet [3, 4]. Das EU-Gesetz [5] ist ehrgeiziger als das amerikanische Vorbild und gilt z.B. auch für seltene Krankheiten. In den USA gingen intensive Gespräche zwischen Industrie, Akademie und Zulassungsbehörden voraus; hier ging die Initiative von EUKommission und EMA aus. Das erste US-Gesetz war freiwillig; die EU drohte von Anfang an mit Nichtzulassung bei Erwachsenen.

\section{Kinderkrebs}

In den 1950er Jahren begannen Onkologen, Kinderkrebs mit Erwachsenen-Zytostatika zu behandeln. So entstand die pädiatrische Onkologie. Chemotherapie zerstört maligne Zellen, aber nicht sehr selektiv. Wir 
sind heute an der Schwelle zu gezielteren Behandlungen. Sind erzwungene Kinderstudien für jedes neue Medikament gegen Erwachsenenkrebs ein Weg vorwärts, oder ist dies mechanisches Kopieren eines Ansatzes, der vor 50 Jahren gut war?

\section{Der medizinische Wert der PIPs}

Die Ergebnisse der Prüfung von PIP-geforderten Studien sind niederschmetternd. Ohne Priorisierung werden für jedes neue Medikament Studien bei Kindern und Jugendlichen verlangt. Aber maligne Erkrankungen bei Kindern sind selten. Imatinib (Glivec) war der erste Tyrosinkinase-Inhibitor (TKI) gegen chronische myeloische Leukämie (CML), die es auch sehr selten bei Kindern gibt. CML ist bei Erwachsenen häufig, und es gibt Nachfolgepräparate für Imatinib (Bosutinib, Nilotinib, Ponatinib). Es gibt nicht genug Kinder für separate Studien für alle TKIs. Aber Firmen müssen sich zu solchen Studien verpflichten, sonst verweigert die EMA die Erwachsenenzulassung [6]. Es gibt jetzt neun MelanomPIPs, und fünf Firmen suchen weltweit Kinder mit metastasiertem Melanom für klinische Studien. Für solche Kinderstudien, die wohl nie genügend rekrutieren werden, wurde der Begriff «Geisterstudie» (ghost study) geprägt, und für die unglückseligen Patienten «therapeutische Geiseln» - in Anlehnung an Shirkeys Wortprägung [7, 8]. Detaillierte Analysen wurden zu Melanom [7], Leukämie [6], zystischer Fibrose [9], multipler Sklerose [10], und Allergenprodukten [4] publiziert.

\section{Drohender Tsunami}

Nicht alle Krankheiten sind bei Kindern selten, z.B. allergische Rhinitis. Die Allergenprodukte für Spezifische Immuntherapie (SIT) sind einen Sonderweg gegangen. Zunächst aus der Natur gewonnen, wurden mit der Zeit auch Allergene industriell prozessiert. Die FDA setzte in den 1980ern eine Standardisierung durch. Die EU klassifizierte sie 1989 als Medikamente, aber duldete weiter named-patient-Gebrauch. 2008 erliess Deutschland ein Gesetz, das die Neuregistrierung von SIT-Produkten vorschrieb [3]. Dafür musste jeweils ein PIP eingereicht werden, insgesamt über 100. Das Paul-Ehrlich-Institut (PEI) und EMA/PDCO entwickelten gemeinsam einen Standard-PIP [11, 12]: für jedes Produkt die gleichen Studien nach dem selben Design, zunächst an Erwachsenen, dann an Kindern und Jugendlichen, mehrere hundert pro Studie und Produkt. Nach der Erwachsenenstudie können Hersteller für Kinder ein Referenzprodukt bestimmen. Das reduziert die verlangten Fünf-Jahres-Studien von 118 auf 58 [13]. Eine gerade laufende SIT-Langzeitstudie plant 800-1000 Kinder - eine Studie. Für die verbleibenden 57 Studien errechnen sich bei jeweils einigen hundert Teilnehmern insgesamt zehntausende Kinder und Jugendliche. Geforderter Schluss der letzten Studien ist Ende 2031 (!) [4]. Die Begründungen sind teils formal (nicht entsprechend den EU-Direktiven zugelassen), teils pseudowissenschaftlich: Das kindliche Immunsystem könnte besser als beim Erwachsenen funktionieren, es könnte Sicherheits-Fragen geben. Aber für Sicherheit wären Behandlungsregister ausreichend. Hersteller müssen bis 2031 entweder zehntausende Kinder in sinnlose Studien rekrutieren, oder sie müssen den Markt verlassen. Diese Studien verhindern in der Plazebo-Gruppe wirksame Behandlung, und das mögliche Fortschreiten zu Asthma wird kaltherzig in Kauf genommen. Die Schweiz erlaubte bei Allergenprodukten die problemlose Nachregistrierung [14]. Niemand ist in der Schweiz gestorben, weil Medikamente nicht EU-Vorgaben entsprachen.

\section{Geistige Grundlagen der EU-Gesetzgebung}

Dokumente, die die EU-Gesetzgebung vorbereiteten [15] und begleiteten [16], enthalten die Forderung nach Evaluation von Medikamenten für Kinder. Aber sie differenzieren nicht zwischen notwendig und nicht notwendig und erwähnen nirgends die pädiatrische Onkologie. Sollten wir Kinder sterben lassen, weil Medikamente nicht für jeden Kinderkrebs zugelassen sind? Die amerikanische Akademie für Kinderheilkunde akzeptiert off-label bei Kindern da, wo sie Sinn macht [17]. Die EU verdreht hingegen berechtigte Bedenken, die zur amerikanischen Gesetzgebung führten, in eine dogmatische hemmungslose Forderung nach möglichst vielen klinischen Studien an Kindern und Jugendlichen.

\section{Erwachsenwerden ist eine juristische, keine biologische Grenze}

Es gibt in diesem Bereich Verunsicherung. Statt konstruktiver Arbeit nutzen die EU-Behörden diese Verunsicherung zur Schaffung eines Betätigungsfelds für enthusiastischen Aktivismus.

\section{Medizinische Experimente am Menschen}

Medizinische Experimente am Menschen sind komplex und heikel. Ein Eingriff in den Körper im Rahmen eines Versuches kann katastrophale Folgen haben. Umgekehrt wären eine halbe Million Menschen in Europa, die als Kinder Krebs hatten, ohne pädiatrische Onkologie nicht mehr am Leben. Verbrecherische Experimente im Dritten Reich hatten die Welt erschüttert. 
Lange konnte sich niemand vorstellen, dass auch amerikanische Wissenschaftler unethische Studien durchführen könnten. Aber 1966 publizierte ein mutiger US-Kliniker eine Liste zweifelhafter, in anerkannten Fachzeitschriften veröffentlichter Studien, so das Verpflanzen von Melanomgewebe von der Tochter auf die Mutter, oder die mutwillige Infizierung von geistig behinderten Kindern mit Hepatitis [18]. Auch die in Alabama vom US Public Health Service (PHS) durchgeführte Tuskegee-Studie erlangte traurige Berühmtheit. Sie beobachtete 1932-1972 schwarze Männer mit Syphilis, ohne sie zu behandeln, auch als es Antibiotika gab. Ein nicht involvierter Behördenangestellter versuchte intern die Einstellung zu erreichen. Man liess ihn auflaufen. Der Spuk endete erst, als er zur Presse ging. Jahrzehnte später entschuldigte sich Präsident Clinton [19].

\section{Warum?}

Geltungssucht, Machthunger und Neid auf erfolgreiche Menschen gibt es überall. Es braucht gesellschaftliche Balance, um Auswüchse zu verhindern. Die von Beecher beschriebenen Studien dienten akademischen Karrieren - solange sie niemand kritisch prüfte. Die TuskegeeStudie bot Bürokraten sichere Arbeitsplätze und Ansehen. Die EMA ist ein Konstrukt über den EU-Staaten, formell kontrolliert von Zulassungsbehörden, EU-Kommission und EU-Parlament, aber mit einem Eigenleben. Die PDCO-Mitglieder kommen grösstenteils aus nationalen Zulassungsbehörden, ferner sind es eine Minderheit klinischer Ärzte und einige andere. Dass ohne Medikamente Millionen von Menschen sterben müssten, wird von den meisten Europäern achselzuckend akzeptiert. Aber Berichte über die böse Pharmaindustrie verkaufen sich gut. Medikamente sollten billig, von edlen Professoren entwickelt, nebenwirkungsfrei und ökologisch sein. Behörden, die gegen die böse Industrie und für Kinder «etwas» tun, geniessen den Vertrauensvorschuss der obrigkeitsgläubigen Europäer. Pseudowissenschaft, Sendungsbewusstsein, Jobsicherheit und mangelnde Kontrolle der EMA verbinden sich hier zu einem unheilvollen Gebräu, das am
Ende Kinder und irregeführte Eltern ausbaden müssen. Der Tsunami sinnloser EU-Kinderstudien konnte sich im Stillen entwickeln. Pharmafirmen wollen es sich nicht mit der Behörde verderben, akademische Kliniker interessieren sich wenig für Zulassungsfragen, und ganz haben die meisten europäischen Pädiater noch nicht ihre Illusionen über die EU verloren. Einzelne PIP-Studien-Protokolle erscheinen auf den ersten Blick akzeptabel und werden von den Ethikkommissionen akzeptiert. Aber wer nur einzelne Bäume anschaut, sieht den Wald nicht.

\section{Wohin?}

Die EU gefährdet die Balance, die pharmazeutischen Fortschritt ermöglicht hat. Das Vertrauen der Bevölkerung in klinische Studien mit Kindern droht Schaden zu nehmen, und europäische Forschung wird sich international isolieren. Eine erste Warnung vor PIP-Studien wurde 2015 publiziert [8]. Auftragsforschungsinstitute werden auch in der Schweiz versuchen, Kinder für sinnlose Studien zu rekrutieren. Schweizer Ärzte, Ethikkommissionen und Eltern sollten auf der Hut sein.

\section{Addendum}

Nach der Artikeleinreichung wurden zwei klinische Studien an Jugendlichen mit metastasiertem Melanom abgebrochen aufgrund langsamer Rekrutierung [1, 2]. Dies ist ein zusätzlicher Beleg für die Aktualität der dargelegten Problematik.

1 BRIM-P: A study of Vemurafenib in pediatric patients with stage IIIC or stage IV melanoma harboring BRAFV600 mutations. https://clinicaltrials.gov/ct2/show/NCT01519323

2 Phase 2 study of Ipilimumab in children and adolescents $(12$ to $<18$ Years) with previously treated or untreated, unresectable stage III or stage IV malignant melanoma. https://clinicaltrials.gov/ct2/ show/NCT01696045

Disclosure statement

Der Autor hat nach der klinischen Ausbildung zum Facharzt für Allgemeinmedizin zwanzig Jahre in der Forschung und Entwicklung pharmazeutischer Firmen gearbeitet, davon fünf Jahre als Global Head Pediatrics bei Novartis und fünf Jahre als Global Head Pediatrics bei Roche. Er ist jetzt selbständig und berät Pharmaunternehmen und akademische Institutionen über Fragen der Arzneimittelentwicklung für Kinder. Eine Tochter des Autors ist schwerbehindert mit einer seltenen Krankheit. Für Publikationen in wissenschaftlichen Zeitschriften erhält der Autor keine Zahlungen oder finanzielle Unterstützung. Dies gilt auch für dieses Manuskript. 


\section{Literatur}

1 Shirkey H: Therapeutic Orphans. Pediatrics. 1999 Sep;104(3 Pt 2): 583-4. http://pediatrics.aappublications.org/content/pediatrics/104/Supplement_3/583.full.pdf

2 Kearns GL, Abdel-Rahman SM, Alander SW, Blowey DL, Leeder IS, Kauffman RE.: Developmental pharmacology--drug disposition, action, and therapy in infants and children. $\mathrm{N}$ Engl J Med. 2003 Sep 18;349(12):1157-67.

3 Therapieallergene-Verordnung 2008: www.bgbl.de/xaver/bgbl/ start.xav?start=//*\%5B@attr_id=\%27bgbl108s2177.pdf\%27\%5D\# bgbl_\%2F\%2F*\%5B\%40attr_id\%3D\%27bgbl108s2177. pdf\%27\%5D__1439545547631

4 Rose K \& Kopp MV: Pediatric Investigation Plans (PIPs) for Specific Immunotherapy (SIT): Questionable Contributions To Childhood Health. Pediatr Allergy Immunol. 2015 Oct 23.

5 VERORDNUNG (EG) Nr. 1901/2006 DES EUROPÄISCHEN PARLAMENTS UND DES RATES vom 12. Dezember 2006 über Kinderarzneimittel und zur Änderung der Verordnung (EWG) Nr. 1768/92, der Richtlinien 2001/20/EG und 2001/83/EG sowie der Verordnung (EG) Nr. 726/2004. Link: http://eur-lex.europa.eu/legal-content/ DE/TXT/?uri=uriserv:OJ.L .2006.378.01.0001.01.

DEU\&toc=OJ:L:2006:378:FULL

6 Rose K \& Walson PD: The contributions of the European Medicines Agency and its pediatric committee to the fight against childhoodleukemia Risk Manag Healthc Policy. 2015 Nov 5;8:185-205. https://www.dovepress.com/the-contributions-of-the-europeanmedicines-agency-and-its-pediatric-c-peer-reviewed-fulltextarticle-RMHP

7 Rose K: European Union Pediatric Legislation Jeopardizes Worldwide, Timely Future Advances in the Care of Children With Cancer. Clinical Therapeutics 2014, 36 (2), 163-177 http://www.clinicaltherapeutics.com/article/SO1492918(14)00018-6/pdf

8 Rose K \& Kummer H: A New Ethical Challenge for Institutional Review Boards (IRBs)/Ethics Committees (ECs) in the Assessment of Pediatric Clinical Trials. Children 2015, 2, 198-210 http://www. mdpi.com/2227-9067/2/2/198

9 Rose K \& Spigarelli MG: Cystic Fibrosis Treatment: A Paradigm for New Pediatric Medicines, Globalization of Drug Development and the Role of the European Medicines Agency. Children 2015, 2(1), 108-130; http://www.mdpi.com/2227-9067/2/1/108
10 Rose K \& Mueller T: Children with Multiple Sclerosis Should Not Become Therapeutic Hostages. Therapeutic Advances in Neurological Disorders 2016 (in press)

11 Englert L, May S, Kaul S \& Vieths S: Die Therapieallergene-Verordnung. Hintergrund und Auswirkungen. Bundesgesundheitsbl 2012 55:351-357 http://www.pei.de/SharedDocs/Downloads/ bundesgesundheitsblatt/2012/2012-therapieallergene-verordnung.pdf? blob=publicationFile \&v $=1$

12 EMA 2015: Allergen Standard PIP: EMA/PDCO Standard Paediatric Investigation Plan for Allergen Products for Specific Immunotherapy Revision 4 http://www.ema.europa.eu/docs/en GB/ document_library/Regulatory_and_procedural_guideline/2009/11/WC500015814.pdf

13 Eichler I \& Sala Soriano E: Close collaboration between academia, industry and drug regulators is required in the development of allergen products for specific immunotherapy in children. Allergy 2011, 66: 999-1004 http://onlinelibrary.wiley.com/ doi/10.1111/j.1398-9995.2011.02582.x/epdf

14 Swissmedic 2014: AW-Verwaltungsverordnung - Vereinfachte Zulassung von Allergenpräparaten. www.swissmedic.ch/ ZLOOO_00_005d VV

15 European Commission 2002: Better Medicines for ChildrenProposed regulatory actions on Paediatric medicinal products. http://ec.europa.eu/health/files/pharmacos/docs/doc2002/feb/ cd_pediatrics_en.pdf

16 EMA 2007: The European paediatric initiative: History of the Paediatric Regulation. http://www.ema.europa.eu/docs/en GB/ document_library/Other/2009/09/WC500003693.pdf

17 Fratarelli DA, Galinkin JL, Green TP, Johnson TD, Neville KA, Paul IM et al.: Off-label use of drugs in children. Pediatrics. 2014 Mar;133(3):563-7 http://pediatrics.aappublications.org/ content/pediatrics/133/3/563.full.pdf

18 Beecher HK. Ethics and clinical research. N Engl J Med 1966; 274 (24): 1354-60

19 Mays VM: The Legacy of the U. S. Public Health Services Study of Untreated Syphilis in African American Men at Tuskegee on the Affordable Care Act and Health Care Reform Fifteen Years After President Clinton's Apology. Ethics Behav. 2012 November 1; 22(6): 411-418. http://www.ncbi.nlm.nih.gov/pmc/articles/PMC3636721/ pdf/nihms458524.pdf 